\title{
High-frame-rate Imaging of a Carotid Bifurcation using a Low-complexity Velocity
} Estimation Approach

di lanni, Tommaso; Villagómez Hoyos, Carlos Armando; Ewertsen, Caroline; Nielsen, Michael Bachmann; Jensen, Jørgen Arendt

\section{Published in:}

Proceedings of 2017 IEEE International Ultrasonics Symposium (IUS)

Publication date:

2017

Document Version

Peer reviewed version

Link back to DTU Orbit

Citation (APA):

di lanni, T., Villagómez Hoyos, C. A., Ewertsen, C., Nielsen, M. B., \& Jensen, J. A. (2017). High-frame-rate Imaging of a Carotid Bifurcation using a Low-complexity Velocity Esstimation Approach. In Proceedings of 2017 IEEE International Ultrasonics Symposium (IUS) IEEE.

\section{General rights}

Copyright and moral rights for the publications made accessible in the public portal are retained by the authors and/or other copyright owners and it is a condition of accessing publications that users recognise and abide by the legal requirements associated with these rights.

- Users may download and print one copy of any publication from the public portal for the purpose of private study or research.

- You may not further distribute the material or use it for any profit-making activity or commercial gain

- You may freely distribute the URL identifying the publication in the public portal 


\title{
High-frame-rate Imaging of a Carotid Bifurcation using a Low-complexity Velocity Estimation Approach
}

\author{
Tommaso Di Ianni ${ }^{1}$, Carlos Armando Villagómez Hoyos ${ }^{1}$, Caroline Ewertsen ${ }^{2}$, Michael Bachmann Nielsen ${ }^{2}$, \\ Jørgen Arendt Jensen ${ }^{1}$ \\ ${ }^{1}$ Center for Fast Ultrasound Imaging, Department of Electrical Engineering, \\ Technical University of Denmark, DK-2800 Lyngby, Denmark \\ ${ }^{2}$ Department of Radiology, Rigshospitalet, Copenhagen University Hospital, Copenhagen 2100, Denmark
}

\begin{abstract}
In this paper, a 2-D vector flow imaging (VFI) method developed by combining synthetic aperture sequential beamforming and directional transverse oscillation is used to image a carotid bifurcation. Ninety-six beamformed lines are sent from the probe to the host system for each VFI frame, enabling the possibility of wireless transmission. The velocity is estimated using a relatively inexpensive 2 -D phase-shift approach, and real-time performance can be achieved in mobile devices. However, high-frame-rate velocities can be obtained by sending the data to a cluster of computers. The objective of this study is to demonstrate the scalability of the method's performance according to the needs of the user and the processing capabilities of the host system. In vivo measurements of a carotid bifurcation of a 54-year-old volunteer were conducted using a linear array transducer connected to the SARUS scanner. The velocities were estimated at a rate of 134 independent frames per second (FPS) to reveal complex flow patterns. A peak frame rate of 2140 FPS can be obtained by generating the images recursively. VFI images are shown during the systolic phase revealing the formation of a vortex in the internal carotid artery. The peak systolic velocity from a range gate in the common tract was $0.76 \mathrm{~m} \mathrm{~s}^{-1}$ with a standard deviation (SD) of $6.1 \%$. The mean velocity profile was calculated from the same range gate with an average SD of $7.86 \%$.
\end{abstract}

\section{INTRODUCTION}

A number of pocket-size ultrasound devices are today available on the market with anatomical and functional imaging features and a form factor such as to fit a lab coat pocket. These systems have enabled the possibility of diagnostic ultrasound imaging at the point of care, allowing a more prompt assessment of the patient's condition. Several studies have demonstrated the value of hand-held ultrasound equipment in a number of medical specialties such as emergency medicine, intensive care and echocardiography [1], [2], as well as in rural districts of low and middle-income countries [3].

App-based systems allow to significantly reduce the cost of the scanner by exploiting general-purpose mobile devices like smartphones or tablets for the processing and visualization. Several attempts have been previously made to integrate flow imaging capabilities in such systems [4], [5], and color flow mapping is currently accessible in most of the commercial scanners. However, these implementations are unable to estimate the velocity in multiple directions and, thus, require a prior knowledge of the flow angle.

Vector flow imaging (VFI) overcomes the flow angle limitations by estimating the velocity components along the lateral and axial directions. Several VFI approaches have been comprehensively investigated, including vector Doppler [6], speckle tracking [7], and transverse oscillation (TO) [8]. The integration of VFI in a portable scanner has the potential to improve the clinical workflow, as quantitative flow measurements are made available with limited operator interaction. Furthermore, by using synthetic aperture (SA) techniques, the velocity field can be accessed everywhere at any time with a high temporal resolution, and velocity profiles can be visualized in a multi-gated approach similar to that previously shown in [9].

A 2-D VFI method has been recently proposed [10], which combines SA sequential beamforming (SASB) [11] and directional TO (DTO) [12]. The method enables wireless transmission of the ultrasound data along with a relatively inexpensive 2-D velocity estimation. In the accompanying paper [13], the method is implemented on a commercially available tablet attaining processing performance suitable for real-time imaging. Nevertheless, the maximum frame rate of 26 frames per second (FPS) is given by the processing capabilities of the device rather than the acquisition sequence.

In this work, the same VFI approach is used to image a carotid bifurcation in vivo with a high frame rate revealing complex flow patterns in the lumen. The objective is to demonstrate the possibility of implementing a flexible imaging framework with scalable performance according to the needs of the operator and the computing capabilities of the host system.

\section{BACKGROUND AND THEORY}

The SASB approach uses a dual-stage beamforming process to lower the datarate between the probe and the processing unit. The received data are initially focused by a fixedfocus beamformer to emulate a virtual source (VS), and the 
TABLE I

MEASUREMENT SETUP AND PROCESSING PARAMETERS

\begin{tabular}{|c|c|c|c|}
\hline Parameter & Flow & B-mode & Unit \\
\hline Transducer & & \\
\hline Array type & & & - \\
\hline Element pitch & \multicolumn{2}{|c|}{0.2} & $\mathrm{~mm}$ \\
\hline Element height & \multicolumn{2}{|c|}{6} & $\mathrm{~mm}$ \\
\hline Number of elements & \multicolumn{2}{|c|}{192} & - \\
\hline Elevation focus & \multicolumn{2}{|c|}{38} & $\mathrm{~mm}$ \\
\hline Center frequency & \multicolumn{2}{|c|}{4.1} & $\mathrm{MHz}$ \\
\hline \multicolumn{4}{|l|}{ Imaging setup } \\
\hline Excitation & 4 cycles & 2 cycles & - \\
\hline Center frequency - $f_{0}$ & \multicolumn{2}{|c|}{4.1} & $\mathrm{MHz}$ \\
\hline $\mathrm{Tx} / \mathrm{Rx}$ apodization & \multicolumn{2}{|c|}{ Tukey $(\alpha=0.6)$} & - \\
\hline VS axial position & -15 & -30 & $\mathrm{~mm}$ \\
\hline Active elements - $L$ & 64 & 96 & - \\
\hline f-number - f\# & -1.17 & -1.56 & - \\
\hline Number of VSs & $K=6$ & 64 & - \\
\hline Distance between VAs - $D$ & 48 & - & elements \\
\hline Pulse repetition freq. - $P R F$ : & \multicolumn{2}{|c|}{15} & $\mathrm{kHz}$ \\
\hline \multicolumn{4}{|l|}{ Processing } \\
\hline Lateral sampling interval & \multicolumn{2}{|c|}{0.1} & $\mathrm{~mm}$ \\
\hline Axial sampling frequency & \multicolumn{2}{|c|}{35} & $\mathrm{MHz}$ \\
\hline Lateral signal length - $M$ & 32 & - & - \\
\hline Number of HRIs - $N$ & 16 & - & - \\
\hline
\end{tabular}

beamformer can be integrated into the probe handle [14]. One single beamformed line, referred to as low-resolution line (LRL), is obtained for each emission. The LRLs are used by a second-stage beamformer to create a high-resolution image (HRI) after $K$ emissions. Therefore, only $K$ lines per HRI must be sent to the host system.

A TO is obtained in the HRIs due to the spatial distribution of the VSs. These are divided in two virtual apertures (VAs) laterally separated by a distance $D$. The TO is used to estimate the velocity in both the lateral and axial directions from $N$ HRIs using a relatively inexpensive 2-D phase-shift estimator [12]. For further details on the method and implementation, readers are referred to [10].

\section{METHODS}

A duplex sequence was designed with the parameters in Table I. A linear array of transducers was connected to the SARUS scanner [15] for the acquisition of B-mode and flow data. A sequence of $9.5 \mathrm{~s}$ of data was saved from the left carotid bifurcation of a 54-year-old female volunteer with no history of cardiovascular disease. The acquisition was performed by an experienced radiologist with the volunteer in supine position. The volunteer was asked to rest for approximately $10 \mathrm{~min}$ to ensure more stationary flow conditions.

The VAs were positioned behind the transducer and steered toward the center of the image with an angle of $\pm 2.5^{\circ}$. Continuous data acquisition was achieved by transmitting one B-mode pulse after every $K=6$ flow emissions, with an effective pulse repetition frequency $P R F_{\text {eff }}=P R F /(K+1)$.

All the processing was carried out off-line in Matlab (The MathWorks, Inc., Natick, MA, USA) using in-house developed code. The received channel data were initially beamformed using a fixed focus to create the LRLs, which were sent to the second beamformer to obtain the HRIs. The beamformation was performed using the BFT3 toolbox [16]. After clutter filtration [17], $N=16$ HRIs were used for the velocity estimation. Therefore, only $16 \times 6=96$ LRLs must be sent from the probe to the host per VFI frame. The velocity estimation was performed in a high-performance computing cluster with a frame rate of 350 FPS. However, according to the emission sequence, only $P R F_{\text {eff }} / N=134 \mathrm{FPS}$ were fully independent. A peak frame rate of $2140 \mathrm{FPS}$ can be achieved by generating the images recursively. The velocities were filtered using a median filter in a temporal window of $25 \mathrm{~ms}$ and a spatial window of $1 \times 1 \mathrm{~mm}^{2}$.

The frame rate of the B-mode sequence was 33 FPS. A binary mask was generated from the B-mode images to discriminate the vessel area from the surrounding tissue. The video was paced down by a factor 14 .

\section{RESUlTS}

A VFI frame is shown in Fig. 1a during the systolic phase. B-mode and VFI images are overlapped. The arrows show the local velocity vectors, while the underlying colors encode the velocity magnitude and direction as specified by the color wheel at the bottom of the image. Fig. 1b displays the velocity magnitude as a function of the time at the locations A and B in the left figure. Point A was selected in the common tract and $B$ in the internal carotid artery (ICA). The mean peak-systolic velocity (PSV) calculated in A from the 10 cardiac cycles was $0.76 \mathrm{~m} \mathrm{~s}^{-1}$ with a SD of $6.1 \%$. The end-diastolic velocity was calculated by averaging the velocities in the period between $70 \%$ and $90 \%$ of the cardiac cycle, and the mean over the 10 cycles was $0.15 \mathrm{~m} \mathrm{~s}^{-1}$. The SD was calculated relative to the mean EDV and was equal to $12.3 \%$. The mean PSV in B was $0.77 \mathrm{~m} \mathrm{~s}^{-1}$ with a SD of $9 \%$. A more turbulent flow and the occurrence of out-of-plane motion can be the reasons for the slightly higher SD registered at the ICA. The velocity field is available at any time everywhere in the image, and multiple profiles like the ones in Fig. $1 \mathrm{~b}$ can be obtained in a multi-gated approach without the need for any additional manual adjustments by the operator. In addition, this can be done retrospectively without acquiring new data.

A low-velocity vortex was identified in the bulb of the ICA and is displayed in Fig. 2 from the same VFI frame in Fig. 1a inside the red rectangle. The vortex is consistent with the findings in previous studies [18].

In Fig. 3, the mean velocity profile is shown in red and the SD displayed as the shadowed area. The SD was calculated by aligning the 10 cardiac cycles in point $\mathrm{A}$ in Fig. 1b, and the averaged relative value over one cycle was $7.86 \%$.

A VFI frame during late systole is displayed in Fig. 4. No vortex is identified in the carotid bulb, and a more parabolic profile is observed in the common tract.

\section{DISCUSSION AND CONCLUSIONS}

In this paper, a 2-D VFI method previously developed for wireless ultrasound combining SASB and DTO was used 


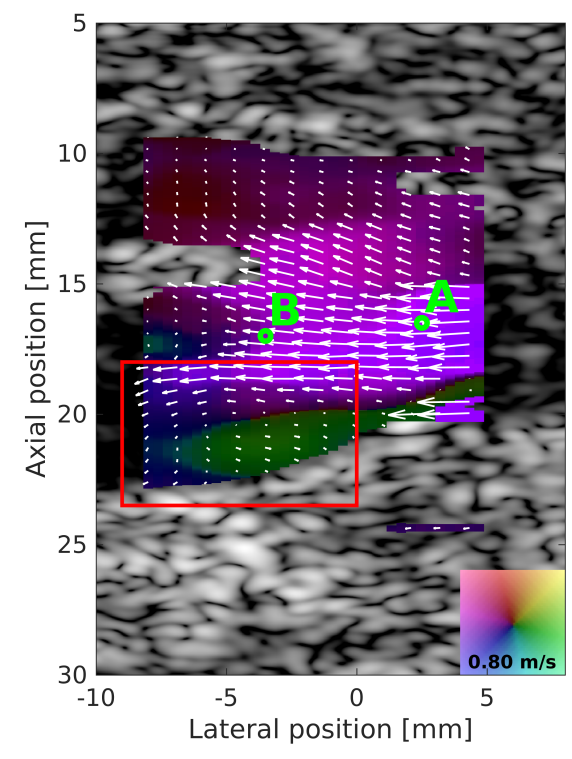

(a)
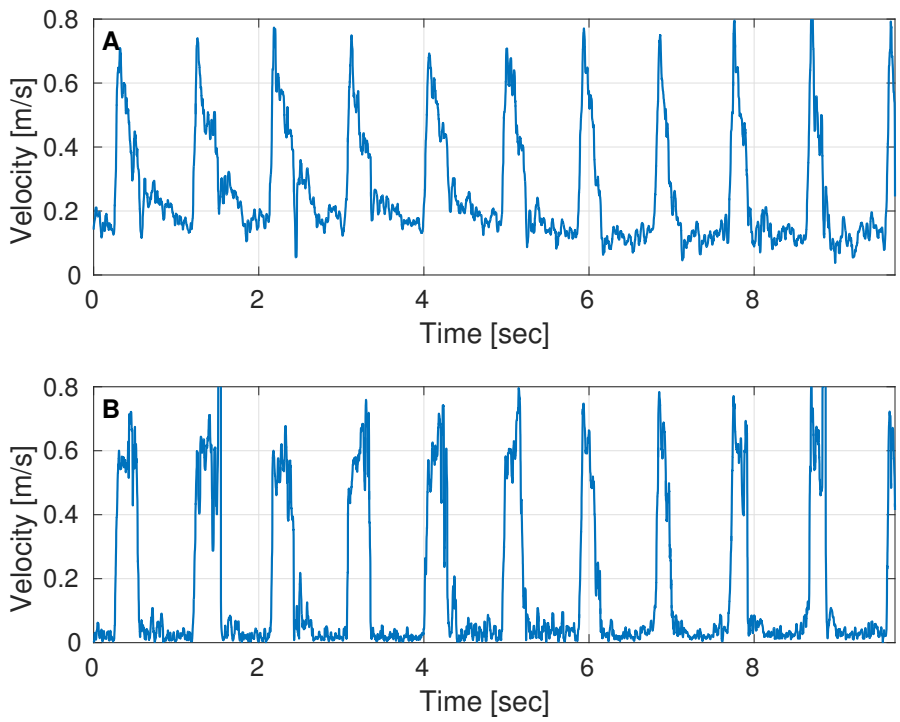

(b)

Fig. 1. (a) VFI frame during the peak systolic phase. A low-velocity vortex is observed in the bulb of the ICA; (b) velocity magnitude as a function of the time in the points A (top) and B (bottom) specified in (a). The velocity is shown for 10 cardiac cycles.

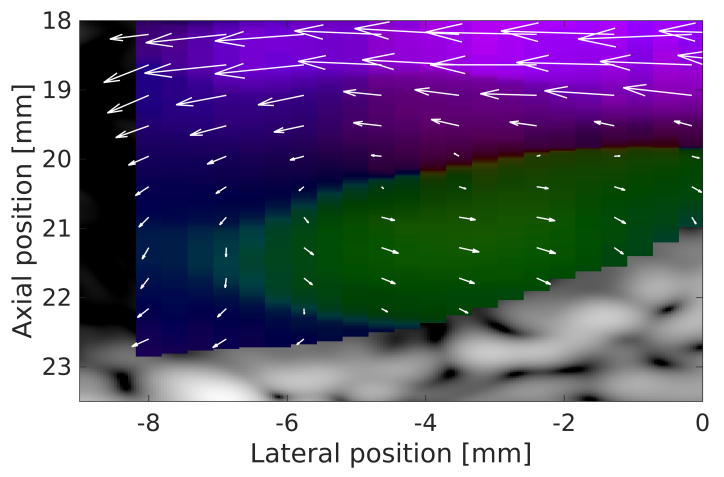

Fig. 2. Vortex formed in the ICA inside the red rectangle in Fig. 1a.

to visualize a carotid bifurcation in vivo. A frame rate of 134 independent frames per second was achieved, which was sufficient to reveal the presence of complex and fast flow dynamics in the carotid bifurcation. The velocity profiles were shown in the common carotid artery and ICA with SDs between 6 and $9 \%$.

The formation of vortices and turbulence is associated with the occurrence of atherosclerotic lesions, therefore the visualization of these phenomena has the potential to lead to an early identification of atherosclerotic sites and allow for preventive intervention. The availability of the velocity field, in addition, opens new possibilities for derived hemodynamic measures like the wall shear stress [19].

The method enables the integration of VFI features in a

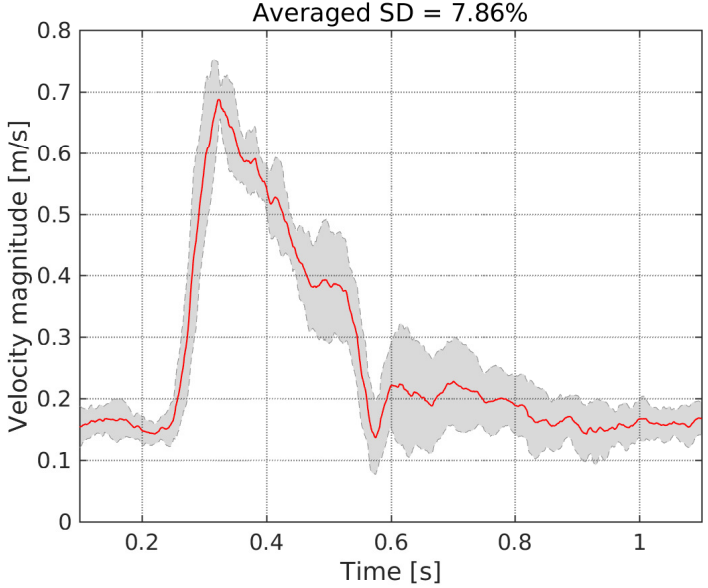

Fig. 3. Mean velocity profile (read) and SD (shadowed area) calculated by aligning the 10 cardiac cycles relative to point $\mathrm{A}$ in Fig. $1 \mathrm{~b}$. The relative SD averaged over one cycle was $7.86 \%$.

hand-held system. At the frame rate of 134 FPS and with the same imaging parameters of [13], a datarate of $66 \mathrm{MB} / \mathrm{s}$ is needed for the transmission of the LRLs to the processing unit.

In conclusion, a highly flexible framework can be implemented, where real-time processing is firstly performed in the host device (tablet), and more complex and advanced imaging modalities can be obtained on-demand by sending the same data to a third processing system. This can also be done retrospectively by storing the full dataset into the memory. 


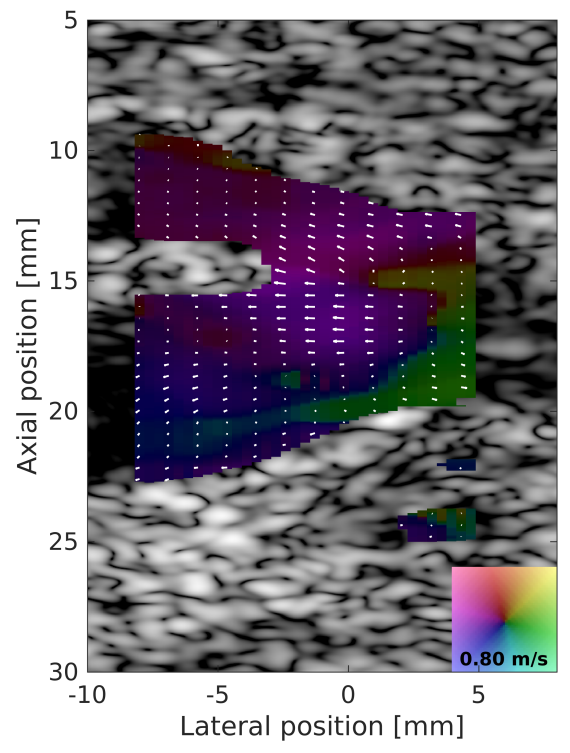

Fig. 4. VFI frame during late systole. No vortex is identified in the carotid bulb and a more parabolic profile is observed in the common tract.

\section{ACKNOWLEDGEMENT}

This work was supported by grant 82-2012-4 from the Danish National Advanced Technology Foundation and by BK Ultrasound.

\section{REFERENCES}

[1] A. W. Kirkpatrick, M. Sirois, K. B. Laupland, D. Liu, K. Rowan, C. G. Ball, S. M. Hameed, R. Brown, R. Simons, S. A. Dulchavsky, D. R. Hamiilton, and S. Nicolaou, "Hand-held thoracic sonography for detecting post-traumatic pneumothoraces: the extended focused assessment with sonography for trauma (EFAST)," J. Trauma, vol. 57, no. 2, pp. 288-295, 2004.

[2] C. Prinz and J. U. Voigt, "Diagnostic accuracy of a hand-held ultrasound scanner in routine patients referred for echocardiography," J. Am. Soc. Echocardiog., vol. 24, no. 2, pp. 111-116, 2011.

[3] S. Sippel, K. Muruganandan, A. Levine, and S. Shah, "Review article: Use of ultrasound in the developing world," Int. J. Emerg. Med., vol. 72, no. 4, December 2011.

[4] M. C. Hemmsen, L. Lassen, T. Kjeldsen, J. Mosegaard, and J. A. Jensen, "Implementation of real-time duplex synthetic aperture ultrasonography," in Proc. IEEE Ultrason. Symp., 2015, pp. 1-4.

[5] E. Jeong, S. Bae, M. Park, W. Jung, J. Kang, and T. Song, "Color Doppler imaging on a smartphone-based portable US system: Preliminary study," in Proc. IEEE Ultrason. Symp., 2015, pp. 1-4.

[6] B. Dunmire, K. W. Beach, K.-H. Labs., M. Plett, and D. E. Strandness, "Cross-beam vector Doppler ultrasound for angle independent velocity measurements," Ultrasound Med. Biol., vol. 26, pp. 1213-1235, 2000.

[7] G. E. Trahey, J. W. Allison, and O. T. von Ramm, "Angle independent ultrasonic detection of blood flow," IEEE Trans. Biomed. Eng., vol. BME-34, no. 12, pp. 965-967, 1987.

[8] J. A. Jensen and P. Munk, "A new method for estimation of velocity vectors," IEEE Trans. Ultrason., Ferroelec., Freq. Contr., vol. 45, no. 3, pp. 837-851, 1998.

[9] S. Ricci, L. Bassi, and P. Tortoli, "Real-time vector velocity assessment through multigate Doppler and plane waves," IEEE Trans. Ultrason., Ferroelec., Freq. Contr., vol. 61, no. 2, pp. 314-324, 2014.

[10] T. Di Ianni, C. Villagomez-Hoyos, C. Ewertsen, T. Kjeldsen, J. Mosegaard, and J. A. Jensen, "A vector flow imaging method for portable ultrasound using synthetic aperture sequential beamforming," IEEE Trans. Ultrason., Ferroelec., Freq. Contr., 2017.
[11] J. Kortbek, J. A. Jensen, and K. L. Gammelmark, "Sequential beamforming for synthetic aperture imaging," Ultrasonics, vol. 53, no. 1, pp. $1-16,2013$.

[12] J. A. Jensen, "Directional transverse oscillation vector flow estimation," IEEE Trans. Ultrason., Ferroelec., Freq. Contr., vol. 64, no. 8, pp. 1194 1204, 2017.

[13] T. Di Ianni, T. Kjeldsen, C. Villagomez-Hoyos, J. Mosegaard, and J. A. Jensen, "Real-time implementation of synthetic aperture vector flow imaging in a consumer-level tablet," in Proc. IEEE Ultrason. Symp., 2017, pp. 1-4.

[14] T. Di Ianni, M. C. Hemmsen, P. L. Muntal, I. H. Jørgensen, and J. A. Jensen, "System-level design of an integrated receiver front end for a wireless ultrasound probe," IEEE Trans. Ultrason., Ferroelec., Freq. Contr., vol. 63, no. 11, pp. 1935-1946, 2016.

[15] J. A. Jensen, H. Holten-Lund, R. T. Nilsson, M. Hansen, U. D. Larsen, R. P. Domsten, B. G. Tomov, M. B. Stuart, S. I. Nikolov, M. J. Pihl, Y. Du, J. H. Rasmussen, and M. F. Rasmussen, "Sarus: A synthetic aperture real-time ultrasound system," IEEE Trans. Ultrason., Ferroelec. Freq. Contr., vol. 60, no. 9, pp. 1838-1852, September 2013.

[16] J. M. Hansen, M. C. Hemmsen, and J. A. Jensen, "An object-oriented multi-threaded software beamformation toolbox," in Proc. SPIE Med. Imag., vol. 7968, March 2011, pp. 79680Y-1-79680Y-9.

[17] C. A. Villagomez-Hoyos, J. Jensen, C. Ewertsen, K. L. Hansen, M. B. Nielsen, and J. A. Jensen, "Energy based clutter filtering for vector flow imaging," in Proc. IEEE Ultrason. Symp., 2017, pp. 1-4.

[18] K. L. Hansen, J. Udesen, F. Gran, J. A. Jensen, and M. B. Nielsen, "Fast blood vector velocity imaging using ultrasound, in-vivo examples of complex blood flow in the vascular system," in Proc. IEEE Ultrason. Symp., 2008, pp. 1068-1071.

[19] C. Poelma, R. M. E. van der Mijle, J. M. Mari, M. X. Tang, P. D. Weinberg, and J. Westerweel, "Ultrasound imaging velocimetry: toward reliable wall shear stress measurements," European Journal of Mechanics - B/Fluids, vol. 35, pp. 70-75, 2012. 